\title{
Nonlinear mirror modes in the presence of hot electrons
}

\author{
E.A. Kuznetsov ${ }^{(a, b)}$, T. Passot ${ }^{(c)}$ and P.L. Sulem ${ }^{(c)}$ \\ ${ }^{(a)}$ P.N. Lebedev Physical Institute RAS, \\ 53 Leninsky Ave., 119991 Moscow, Russia \\ (b) Space Research Institute RAS, 84/32 Profsoyuznaya str., 117997, Moscow, Russia \\ (c) Université de Nice-Sophia Antipolis, \\ CNRS, Observatoire de la Côte d'Azur, \\ PB 4229, 06304 Nice Cedex 4, France
}

\begin{abstract}
A non-perturbative calculation of the gyrotropic pressures associated with large-scale mirror modes is performed, taking into account a finite, possibly anisotropic electron temperature. In the small-amplitude limit, this leads to an extension of an asymptotic model previously derived for cold electrons. A model equation for the profile of subcritical finite-amplitude large-scale structures is also presented.
\end{abstract}


PACS: 52.35.Py, 52.25.Xz, 94.30.cj, 94.05.-a

\section{INTRODUCTION}

Pressure-balanced magnetic structures in the form of strong magnetic enhancements (humps) and depressions (holes) that are quasi-stationary in the plasma frame, with no or little change in the magnetic field direction, are commonly observed in regions of the solar wind and of planetary magnetosheaths with relatively large $\beta$ and a dominant (generally ion) temperature in the transverse direction ( see, for instance, [1, 2] and references therein). The origin of these structures is still not fully understood, but they are usually viewed as nonlinearly saturated states of the mirror instability (MI) discovered by Vedenov and Sagdeev [3]. It is a kinetic instability whose growth rate was first obtained under the assumption of cold electrons, a regime where the contributions of the parallel electric field $E_{\|}$ can be neglected. However, in realistic space plasmas, the electron temperature

can hardly be ignored [4]. The linear theory retaining the electron temperature and its possible anisotropy, in the quasi-hydrodynamic limit (which neglects finite Larmor radius corrections), was developed in the case of bi-Maxwellian distribution functions by several authors [5]-[9]. A general estimate of the growth rate under the sole condition that it is small compared with the ion gyrofrequency (a condition reflecting close vicinity to threshold) is presented in [10]. The instability then develops in quasi-perpendicular directions, making the parallel magnetic perturbation dominant. This analysis includes in particular regimes with a significant electron temperature anisotropy for which the instability extends beyond the ion Larmor radius. In the limit where the instability is limited to scales large compared with the ion Larmor radius, only the leading order contribution in terms of the small parameter $\gamma /\left(|k|_{z} v_{\| i}\right)$ is to be retained in estimating Landau damping, and 
the growth rate is given by

$$
\begin{aligned}
\gamma= & \frac{2}{\sqrt{\pi}} \frac{T_{\| i}}{T_{\perp i}} \frac{\left|k_{z}\right| v_{\| i}}{E}\left\{\Gamma-\frac{1}{\beta_{\perp}}\left(1+\frac{\beta_{\perp}-\beta_{\|}}{2}\right) \frac{k_{z}^{2}}{k_{\perp}^{2}}\right. \\
& \left.-\frac{3}{4\left(1+\theta_{\perp}\right)}\left(\frac{T_{\perp i}}{T_{\| i}}-1\right)(1+F) k_{\perp}^{2} r_{L}^{2}\right\},
\end{aligned}
$$

where

$$
\Gamma=\frac{T_{\perp i}}{T_{\| i}} \frac{\left(\theta_{\|}+\theta_{\perp}\right)^{2}+2 \theta_{\|}\left(\theta_{\perp}^{2}+1\right)}{2 \theta_{\|}\left(1+\theta_{\perp}\right)\left(\theta_{\|}+1\right)}-1-\frac{1}{\beta_{\perp}}
$$

measures the distance to threshold and

$$
\begin{aligned}
E= & \frac{1+\theta_{\perp}}{\left(1+\theta_{\|}\right)^{2}}\left[2+\theta_{\perp}\left(4+\theta_{\perp}\right)+\theta_{\|}^{2}\right] \\
F= & \frac{T_{\| e}}{T_{\| e}+T_{\| i}}\left\{-1+\frac{\theta_{\perp}}{\theta_{\|}}\right. \\
& \left.-\frac{2}{3} \frac{T_{\| i}}{T_{\perp i}}\left[\left(\frac{T_{\| i}}{T_{\perp i}}-1\right) \frac{1}{\beta_{\perp i}}-\theta_{\perp}\left(\frac{T_{\perp e}}{T_{\| e}}-1\right)\right]\right\} .
\end{aligned}
$$

Here, $T_{\perp \alpha}$ and $T_{\| \alpha}$ are the perpendicular and parallel (relative to the ambient magnetic field $\mathbf{B}_{0}$ taken in the $z$ direction) temperatures of the species $\alpha(\alpha=$ $i$ for ions and $\alpha=e$ for electrons ), $\theta_{\perp}=T_{\perp e} / T_{\perp i}, \theta_{\|}=T_{\| e} / T_{\| i}$ and $\beta_{\perp}=$ $\beta_{\perp i}+\beta_{\perp e}$ with $\beta_{\perp \alpha}=8 \pi p_{\perp \alpha} / B_{0}^{2}$ where $p_{\perp \alpha}$ is the perpendicular thermal pressure (similar definition for $\beta_{\|}$). Furthermore, the parallel thermal velocity is defined as $v_{\| \alpha}=\sqrt{2 T_{\| \alpha} / m_{\alpha}}$, and $r_{L}=\left(2 T_{\perp i} / m_{p}\right)^{1 / 2} / \Omega_{i}$ denotes the ion Larmor radius $\left(\Omega_{i}=e B_{0} / m_{i} c\right.$ is the ion gyrofrequency).

The growth rate given by Eq. (1) has the same structure as in the cold electron regime considered in [11] in the case of bi-Maxwellian ions and generalized in [12] and [9] to an arbitrary distribution function. The first term within the curly brackets provides the threshold condition which coincides with that given in [5][11]. The second one reflects the magnetic field line elasticity and the third one (where $F$ depends on the electron temperatures due to the coupling between the species induced by the parallel electric field which is relevant for hot electrons) provides the arrest of the instability at small scales by finite Larmor radius (FLR) effects. 
An aim of this letter is to extend to hot electrons the weakly nonlinear analysis previously developed for cold electrons [13, 14]. Since in this asymptotics, FLR contributions appear only at the linear level, the idea is to use the drift kinetic formalism to calculate the nonlinear terms. We show that the equation governing the evolution of weakly nonlinear mirror modes has the same form as in the case of cold electrons. In particular, the sign of the nonlinear coupling coefficient that prescribes the shape of mirror structures, is not changed. This equation is of gradient type equation with a free energy (or a Lyapunov functional) which is unbounded from below. This leads to finite-time blowing-up solutions [15], associated with the existence of a subcritical bifurcation [13, 14]. To describe subcritical stationary mirror structures in the strongly nonlinear regime, we present an anisotropic MHD model where the perpendicular and parallel pressures are determined from the drift kinetic equations in the adiabatic approximation, in the form of prescribed functions of the magnetic field amplitude.

\section{BASIC EQUATIONS}

A main condition characterizing mirror modes, at least near threshold, is provided by the force balance equation

$$
\begin{aligned}
& -\nabla\left(p_{\perp}+\frac{B^{2}}{8 \pi}\right)+\left[1+\frac{4 \pi}{B^{2}}\left(p_{\perp}-p_{\|}\right)\right] \frac{(\mathbf{B} \cdot \nabla) \mathbf{B}}{4 \pi} \\
& +\mathbf{B}(\mathbf{B} \cdot \nabla)\left(\frac{p_{\perp}-p_{\|}}{B^{2}}\right)-\nabla \cdot \boldsymbol{\Pi}=0,
\end{aligned}
$$

where the pressure tensor, viewed as the the sum of the contributions of the various species, has been written as the sum of a gyrotropic part characterized by the parallel $\left(p_{\|}=\sum_{\alpha} p_{\| \alpha}\right)$ and perpendicular $\left(p_{\perp}=\sum_{\alpha} p_{\perp \alpha}\right)$ pressures, and of a gyroviscous contribution $\Pi$ originating from the sole ion FLR effects when concentrating on scales large compared with the electron Larmor radius. As mentioned above, FLR effects arising only at the linear level with respect to the amplitude of the perturbations, the other linear and nonlinear contributions can be evaluated 
from the drift kinetic equation for each particle species

$$
\frac{\partial f_{\alpha}}{\partial t}+v_{\|} \mathbf{b} \cdot \nabla f_{\alpha}+\left[-\mu \mathbf{b} \cdot \nabla B+\frac{e_{\alpha}}{m_{\alpha}} E_{\|}\right] \frac{\partial f_{\alpha}}{\partial v_{\|}}=0
$$

We ignore the transverse electric drift which is subdominant for mirror modes. In this approximation, both ions and electrons move in the direction of the magnetic field (defined by the unit vector $\mathbf{b}=\mathbf{B} / B$ ) under the effect of the magnetic force $\mu \mathbf{b} \cdot \nabla B$ and the parallel electric field $E_{\|}=-\mathbf{b} \cdot \nabla \phi$ where the magnetic moment $\mu=v_{\perp}^{2} /(2 B)$ is an adiabatic invariant which plays the role of a parameter in Eq. (44). Here $\phi$ is the electric potential. The quasi-neutrality condition $n_{e}=n_{i} \equiv n$, where $n_{\alpha}=B \int f_{\alpha} d \mu d v_{\|} d \varphi \equiv \int f_{\alpha} d^{3} v$, is used to close the system and eliminate $E_{\|}$.

In this framework where FLR effects are neglected, the gyrotropic pressures are given by $p_{\alpha \|} \equiv m_{\alpha} \int v_{\|}^{2} f_{\alpha} d^{3} v=m_{\alpha} B \int v_{\|}^{2} f_{\alpha} d \mu d v_{\|} d \varphi$, and $p_{\alpha \perp} \equiv \frac{1}{2} m_{\alpha} \int v_{\perp}^{2} f_{\alpha} d^{3} v=$ $m_{\alpha} B^{2} \int \mu f_{\alpha} d \mu d v_{\|} d \varphi$.

The asymptotic equation governing the mirror dynamics near threshold is obtained by expanding Eqs. (3), (4) and the quasi-neutrality condition, with the pressure tensor elements for each species computed near a bi-Maxwellian equilibrium state characterized by the temperatures $T_{\perp \alpha}$ and $T_{\| \alpha}$.

\section{LINEAR INSTABILITY}

Before turning to the nonlinear regime, we briefly review the derivation of the MI linear growth rate in the simplified framework provided by the drift kinetic approximation which is only valid at scales large enough for FLR effects to be subdominant.

Linearizing Eq. (3) about the background field $\mathbf{B}_{\mathbf{0}}$ and equilibrium pressures $p_{\perp}^{(0)}$ and $p_{\|}^{(0)}$, and considering perturbations $\widetilde{\mathbf{B}}$ and $p_{\perp}^{(1)} \propto e^{-i \omega t+i \mathbf{k} \cdot \mathbf{r}}$, we get

$$
p_{\perp}^{(1)}+\frac{B_{0} \widetilde{B}_{z}}{4 \pi}=-\frac{k_{z}^{2}}{k_{\perp}^{2}}\left(1+\frac{\beta_{\perp}-\beta_{\|}}{2}\right) \frac{B_{0} \widetilde{B}_{z}}{4 \pi} .
$$


Here, $p_{\perp}^{(1)}$ has to be calculated from the linearized drift kinetic equation

$$
\frac{\partial f_{\alpha}^{(1)}}{\partial t}+v_{\|} \frac{\partial f_{\alpha}^{(1)}}{\partial z}+\left[-\mu \frac{\partial \widetilde{B}_{z}}{\partial z}+\frac{e_{\alpha}}{m_{\alpha}} E_{\|}\right] \frac{\partial f_{\alpha}^{(0)}}{\partial v_{\|}}=0
$$

where we assume each $f_{\alpha}^{(0)}$ to be a bi-Maxwellian distribution function

$$
f_{\alpha}^{(0)}=A_{\alpha} \exp \left[-\frac{v_{\|}^{2}}{v_{\| \alpha}^{2}}-\frac{\mu B_{0} m_{\alpha}}{T_{\perp \alpha}}\right],
$$

with $A_{\alpha}=n_{0} m_{\alpha} /\left(2 \pi \sqrt{\pi} v_{\| \alpha} T_{\perp \alpha}\right)$.

Equation (6) is solved in Fourier representation, as

$$
f_{\alpha}^{(1)}=-\frac{\mu \widetilde{B}_{z}+\frac{e_{\alpha}}{m_{\alpha}} \phi}{\omega-k_{z} v_{\|}} k_{z} \frac{\partial f_{\alpha}^{(0)}}{\partial v_{\|}}
$$

The neutrality condition allows one to express the potential $\phi$ in terms of $\widetilde{B}_{z}$. Indeed, assuming $\zeta=\sqrt{\pi} \omega /\left(\left|k_{z}\right| v_{\| i}\right) \ll 1$ (so that the contribution from the Landau pole is small),

$$
\int f_{i}^{(1)} d v_{z} d \mu d \varphi=-\frac{n_{0}}{B_{0} T_{\| i}}\left[T_{\perp i} \frac{\widetilde{B}_{z}}{B_{0}}+e \phi\right][1+i \zeta] .
$$

Similarly, neglecting the electron Landau resonance contribution because of the small mass ratio,

$$
\int f_{e}^{(1)} d v_{\|} d \mu d \varphi=-\frac{n_{0}}{B_{0} T_{\| e}}\left[T_{\perp e} \frac{\widetilde{B}_{z}}{B_{0}}-e \phi\right]
$$

Consequently,

$$
e \phi \approx \frac{T_{\perp i}}{1+\theta_{\|}}\left[\left(\theta_{\perp}-\theta_{\|}\right)-\frac{\theta_{\|}\left(1+\theta_{\perp}\right)}{1+\theta_{\|}} i \zeta\right] \frac{\widetilde{B}_{z}}{B_{0}} .
$$

We thus recover that for mirror modes, the parallel electric field vanishes when the electrons are cold $\left(\theta_{\perp}=\theta_{\|}=0\right)$. Interestingly, when $\theta_{\perp}=\theta_{\|}$, only the Landau pole contributes to $\phi$.

It is now necessary to evaluate

$$
p_{\perp}^{(1)}=2 \frac{\widetilde{B}_{z}}{B_{0}} p_{\perp}^{(0)}+B_{0}^{2} \sum_{\alpha} m_{\alpha} \int \mu f_{\alpha}^{(1)} d \mu d v_{\|} d \varphi .
$$


Using

$$
\begin{aligned}
\int \frac{k_{z} v_{\|}}{\omega-k_{z} v_{\|}} f_{i}^{(0)} d \mu d v_{\|} d \varphi & =-\frac{n_{0}}{B_{0}}(1+i \zeta) \\
\int \frac{k_{z} v_{\|}}{\omega-k_{z} v_{\|}} f_{e}^{(0)} d \mu d v_{\|} d \varphi & =-\frac{n_{0}}{B_{0}}
\end{aligned}
$$

we get

$$
p_{\perp}^{(1)}=-\beta_{\perp} \frac{B_{0}^{2}}{4 \pi}\left[\frac{1}{\beta_{\perp}}+\Gamma+\frac{T_{\perp i}}{T_{\| i}} \frac{i \zeta D}{2\left(1+\theta_{\perp}\right)}\right] \frac{\widetilde{B}_{z}}{B_{0}} .
$$

Substituting this expression into the linearized force balance equation yields the linear instability growth rate given by Eq. (1), up to the FLR term which is not captured by the drift kinetic approximation. Note that the growth rate given by Eq. (1) is consistent with the applicability condition $\gamma /\left|k_{z}\right| \ll v_{\| i}$ near threshold $(\Gamma \ll 1)$, as $k_{z}$ and $\left(k_{z} / k_{\perp}\right)^{2}$ scale like $\Gamma$, while $\gamma$ like $\Gamma^{2}$.

\section{GENERAL PRESSURE ESTIMATES}

As demonstrated in [13, 14], the scalings resulting from the linear theory near threshold imply an adiabaticity condition to leading order. It is thus enough to consider the stationary kinetic equation

$$
v_{\|} \mathbf{b} \cdot \nabla f_{\alpha}-(\mathbf{b} \cdot \nabla)\left[\mu B+\frac{e_{\alpha}}{m_{\alpha}} \phi\right] \frac{\partial f_{\alpha}}{\partial v_{\|}}=0 .
$$

It turns out that Eq. (14) is exactly solvable, the general solution being an arbitrary function $f_{\alpha}=g_{\alpha}\left(\mu, W_{\alpha}\right)$ of the particle energy $W_{\alpha}=v_{\|}^{2} / 2+\mu B+\frac{e_{\alpha}}{m_{\alpha}} \phi$, and of $\mu$. To find the function $g_{\alpha}\left(\mu, W_{\alpha}\right)$, we use the adiabaticity argument which means that, to leading order, $g_{\alpha}$ as a function of $\mu$ and $W_{\alpha}$ retains its form during the evolution. Therefore, the function $g_{\alpha}\left(\mu, W_{\alpha}\right)$ is found by matching with the initial distribution function $f_{\alpha}^{(0)}$ given by Eq. (7) which corresponds to $\phi=0$ and $W_{\alpha}=\frac{v_{\|}^{2}}{2}+\mu B_{0}$. We get

$$
\begin{aligned}
& g_{\alpha}\left(\mu, W_{\alpha}\right)=A_{\alpha} \exp \left[-\frac{v_{\|}^{2}}{v_{\| \alpha}^{2}}-\frac{\mu B_{0} m_{\alpha}}{T_{\perp \alpha}}\right] \\
& \quad=A_{\alpha} \exp \left[-\frac{2 W_{\alpha}}{v_{\| \alpha}^{2}}+\mu B_{0} m_{\alpha}\left(\frac{1}{T_{\| \alpha}}-\frac{1}{T_{\perp \alpha}}\right)\right] .
\end{aligned}
$$


Thus, $g_{\alpha}\left(\mu, W_{\alpha}\right)$ is a Boltzmann distribution function with respect to $W_{\alpha}$ but, at fixed $W_{\alpha}$, it displays an exponential growth relatively to $\mu$ if $T_{\perp \alpha}>T_{\| \alpha}$. This effect can however be compensated by the dependence of $W_{\alpha}$ in $\mu$. This means that only a fraction of the phase space $\left(\mu, W_{\alpha}\right)$ is accessible, a property possibly related with the existence of trapped and untrapped particles.

Note that expanding Eq. (15) relatively to $\widetilde{B}_{z} / B_{0}$ and $e \phi^{(1)} / T_{\perp i}$ reproduces the first order contribution to the distribution function given by Eq. (8) with $\omega=0$, and also the second order correction found in [13, 14] in the case of cold electrons. It should be emphasized that Eq. (15) only assumes adiabaticity and remains valid for finite perturbations.

The function $g_{\alpha}$ can also be rewritten in terms of $v_{\|}, v_{\perp}$ and $\phi$ as

$$
\begin{aligned}
g_{\alpha}= & A_{\alpha} \exp \left[-\frac{m_{\alpha} v_{\|}^{2}}{2 T_{\| \alpha}}-\frac{e_{\alpha} \phi}{T_{\| \alpha}}\right] \times \\
& \exp \left\{-\frac{m_{\alpha} v_{\perp}^{2}}{2 T_{\perp \alpha}}\left(\frac{T_{\perp \alpha}}{T_{\| \alpha}}-\frac{B_{0}}{B}\left[\frac{T_{\perp \alpha}}{T_{\| \alpha}}-1\right]\right)\right\},
\end{aligned}
$$

which can be viewed as the bi-Maxwellian distribution function with the renormalized transverse temperature

$$
T_{\perp \alpha}^{(e f f)}=T_{\perp \alpha}\left[\frac{T_{\perp \alpha}}{T_{\| \alpha}}-\frac{B_{0}}{B}\left(\frac{T_{\perp \alpha}}{T_{\| \alpha}}-1\right)\right]^{-1} .
$$

Note the Boltzmann factor exp $-\left[e_{\alpha} \phi / T_{\| \alpha}\right]$ in the expression of $g_{\alpha}$. For cold electrons, the ion distribution function was obtained in [16] by assuming that it remains bi-Maxwellian, and owing to the invariance of the kinetic energy and of the magnetic moment. This estimate, obtained by neglecting both time dependency (and consequently Landau resonance) and finite Larmor radius corrections, reproduces the closure condition given in [17].

After rewriting Eq. (15) in the form

$$
g_{\alpha}=A_{\alpha} \exp \left[-\frac{e_{\alpha} \phi}{T_{\| \alpha}}-\frac{v_{\|}^{2}}{v_{\| \alpha}^{2}}-\frac{\mu B_{0} m_{\alpha}}{T_{\perp \alpha}}\left(1+\frac{T_{\perp \alpha}}{T_{\| \alpha}} \frac{B-B_{0}}{B_{0}}\right)\right]
$$


the quasi-neutrality condition gives

$$
\begin{aligned}
& \left(1+\frac{T_{\perp i}}{T_{\| i}} \frac{B-B_{0}}{B_{0}}\right)^{-1} \exp \left(-\frac{e \phi}{T_{\| i}}\right)= \\
& \left(1+\frac{T_{\perp e}}{T_{\| e}} \frac{B-B_{0}}{B_{0}}\right)^{-1} \exp \left(\frac{e \phi}{T_{\| e}}\right)
\end{aligned}
$$

or

$$
\begin{aligned}
& e \phi=\left(T_{\| i}^{-1}+T_{\| e}^{-1}\right)^{-1} \times \\
& \log \left[\left(1+\frac{T_{\perp e}}{T_{\| e}} \frac{B-B_{0}}{B_{0}}\right)\left(1+\frac{T_{\perp i}}{T_{\| i}} \frac{B-B_{0}}{B_{0}}\right)^{-1}\right] .
\end{aligned}
$$

Interestingly, the electron density (and thus also that of the ions)

$$
n_{e}=n_{0} \frac{B}{B_{0}}\left(1+\frac{T_{\perp e}}{T_{\| e}} \frac{B-B_{0}}{B_{0}}\right)^{-1} \exp \left[\frac{e \phi}{T_{\| e}}\right]
$$

has the usual Boltzmann factor $\exp \left[e \phi / T_{\| e}\right]$ and also an algebraic prefactor depending on the magnetic field $B$. In the case of isotropic electron temperature $\left(T_{\perp e}=T_{\| e} \equiv T_{e}\right)$, the electron density has the usual Boltzmann form $n_{e}=n_{0} \exp \left[e \phi / T_{e}\right]$.

Equation (18) shows that the potential vanishes in two cases: for cold electrons and also when electron and ion temperature anisotropies $a_{e}$ and $a_{i}$ (with $a_{\alpha}=$ $\left.T_{\perp \alpha} / T_{\| \alpha}\right)$ are equal, a case considered in the linear theory of the mirror instability [5, 11, 18].

In order to evaluate explicitly the perpendicular pressure for each species

$$
\begin{aligned}
& p_{\perp \alpha}=m_{\alpha} B^{2} \int \mu g_{\alpha} d \mu d v_{\|} d \varphi \\
& \quad=n_{0} T_{\perp \alpha} \frac{B^{2}}{B_{0}^{2}}\left(1+\frac{T_{\perp \alpha}}{T_{\| \alpha}} \frac{B-B_{0}}{B_{0}}\right)^{-2} \exp \left(-\frac{e_{\alpha} \phi}{T_{\| \alpha}}\right),
\end{aligned}
$$

where $e \phi$ is given by Eq. (18), it is convenient to introduce the functions

$$
\begin{aligned}
& S_{\perp i}(u)=\left(\frac{1+u}{1+a_{i} u}\right)^{2}\left(\frac{1+a_{i} u}{1+a_{e} u}\right)^{c_{i}} \\
& S_{\perp e}(u)=\left(\frac{1+u}{1+a_{e} u}\right)^{2}\left(\frac{1+a_{e} u}{1+a_{i} u}\right)^{c_{e}},
\end{aligned}
$$


with the notations $u=\left(B-B_{0}\right) / B_{0}$ and $c_{\alpha}=T_{\| \alpha}^{-1} /\left(T_{\| i}^{-1}+T_{\| e}^{-1}\right)$. The two latter functions transform one into the other by exchanging the subscripts $i$ and $e$. The ion and electron perpendicular pressures are then written as $p_{\perp \alpha}=n_{0} T_{\perp \alpha} S_{\perp \alpha}(u)$. In the special case of cold electrons,

$$
p_{\perp}=n_{0} T_{\perp i} \frac{B^{2}}{B_{0}^{2}}\left(1+\frac{T_{\perp i}}{T_{\| i}} \frac{B-B_{0}}{B_{0}}\right)^{-2},
$$

which is algebraic relatively to $B$. From this expression as well as from the general formula for $p_{\perp}=p_{\perp i}+p_{\perp e}$ given by Eqs. (20) and (21) it follows that the perpendicular and magnetic pressures are anticorrelated. When $B$ increases (decreases), the ratio of the perpendicular to the magnetic pressure, i.e. the local $\beta_{\perp}$, decreases (increases), which corresponds to a reduction (an increase) of the distance to threshold. This implies that the instability cannot saturate at small amplitudes.

Similarly, for the parallel pressure, we have

$$
p_{\| \alpha}=n_{0} T_{\| \alpha} \frac{B}{B_{0}}\left(1+\frac{T_{\perp \alpha}}{T_{\| \alpha}} \frac{B-B_{0}}{B_{0}}\right)^{-1} \exp \left(-\frac{e_{\alpha} \phi}{T_{\| \alpha}}\right) .
$$

that rewrites $p_{\| \alpha}=n_{0} T_{\| \alpha} S_{\| \alpha}(u)$ with

$$
\begin{aligned}
& S_{\| i}(u)=\left(\frac{1+u}{1+a_{i} u}\right)\left(\frac{1+a_{i} u}{1+a_{e} u}\right)^{c_{i}} \\
& S_{\| e}(u)=\left(\frac{1+u}{1+a_{e} u}\right)\left(\frac{1+a_{e} u}{1+a_{i} u}\right)^{c_{e}} .
\end{aligned}
$$

\section{THE WEAKLY NONLINEAR REGIME}

As it follows from Eq. (5), in the linear regime near threshold, the fluctuations of perpendicular and magnetic pressures almost compensate each other. In the weakly nonlinear regime, the second order correction to the total (perpendicular plus magnetic) pressure is thus relevant and leads to a local shift of $\Gamma$. To find this correction, we consider the expansions of the perpendicular pressures of the ions and electrons in the $u$ variable. Because of the symmetry between the functions 
$S_{\perp i}(u)$ and $S_{\perp e}(u)$, it is enough to consider the expansion

$$
\begin{aligned}
S_{\perp i}(u)= & 1+u\left(2-2 a_{i}-c_{i}\left(a_{e}-a_{i}\right)\right) \\
& +u^{2}\left[c_{i}\left(a_{e} a_{i}-a_{i}^{2}+\frac{1}{2}\left(a_{e}-a_{i}\right)^{2}\right)\right. \\
& -4 a_{i}+3 a_{i}^{2}+\frac{1}{2} c_{i}^{2}\left(a_{e}-a_{i}\right)^{2}-2 c_{i}\left(a_{e}-a_{i}\right) \\
& \left.+2 \alpha_{i} c_{i}\left(a_{e}-a_{i}\right)+1\right]+O\left(u^{3}\right)
\end{aligned}
$$

As a result, the second order contributions to the perpendicular ion pressure is given by

$$
\begin{aligned}
p_{i \perp}^{(2)} & =n_{0} T_{\perp i}\left[3 a_{i}^{2}-4 a_{i}+1+c_{i}\left(a_{e}-a_{i}\right)\right. \\
& \left.\times\left(\frac{1}{2}\left(c_{i}+1\right)\left(a_{e}-a_{i}\right)-2+3 a_{i}\right)\right] u^{2},
\end{aligned}
$$

with an analogous formula for the perpendicular electron pressure, obtained by exchanging the $i$ and $e$ indices. Furthermore, the threshold condition rewrites

$$
\begin{aligned}
\frac{B_{0}^{2}}{4 \pi}+ & n_{0}\left\{T_{\perp i}\left[2-2 a_{i}-c_{i}\left(a_{e}-a_{i}\right)\right]\right. \\
& \left.+T_{\perp e}\left[2-2 a_{e}+c_{e}\left(a_{e}-a_{i}\right)\right]\right\}=0 .
\end{aligned}
$$

The quadratic contributions to the pressure balance (3), originating from $p_{i \perp}^{(2)}+$ $p_{e \perp}^{(2)}+\left(B-B_{0}\right)^{2} /(8 \pi)$, are collected in a term $\Lambda\left(\frac{B-B_{0}}{B_{0}}\right)^{2}$ with

$$
\begin{aligned}
\Lambda= & n_{0}\left\{T _ { \perp i } \left(3 a_{i}^{2}-4 a_{i}+1\right.\right. \\
& \left.+c_{i}\left(a_{e}-a_{i}\right)\left[\frac{1}{2}\left(1+c_{i}\right)\left(a_{e}-a_{i}\right)-2+3 a_{i}\right]\right) \\
& +T_{\perp e}\left(3 a_{e}^{2}-4 a_{e}+1+c_{e}\left(a_{e}-a_{i}\right)\right. \\
& \left.\left.\times\left[\frac{1}{2}\left(1+c_{e}\right)\left(a_{e}-a_{i}\right)+2-3 a_{e}\right]\right)\right\}+\frac{B_{0}^{2}}{8 \pi} .
\end{aligned}
$$

The value $\Lambda_{c}$ of $\Lambda$ at threshold is obtained by expressing $B_{0}^{2} / 8 \pi$ by means of Eq. (26), which gives

$$
\Lambda_{c}=n_{0}\left\{T _ { \perp i } \left[3 a_{i}^{2}-4 a_{i}+1\right.\right.
$$




$$
\begin{aligned}
& +c_{i}\left(a_{e}-a_{i}\right)\left(\frac{1}{2}\left(1+c_{i}\right)\left(a_{e}-a_{i}\right)-2+3 a_{i}\right) \\
& \left.-\frac{1}{2}\left(2-2 a_{i}-c_{i}\left(a_{e}-a_{i}\right)\right)\right] \\
& +T_{\perp e}\left[3 a_{e}^{2}-4 a_{e}+1+c_{e}\left(a_{e}-a_{i}\right)\right. \\
& \times\left(\frac{1}{2}\left(1+c_{e}\right)\left(a_{e}-a_{i}\right)+2-3 a_{e}\right] \\
& \left.\left.-\frac{1}{2}\left(2-2 a_{e}+c_{e}\left(a_{e}-a_{i}\right)\right)\right]\right\} .
\end{aligned}
$$

After some algebra, defining $\lambda_{c}=\Lambda_{c} /\left(n_{0} T_{\perp i}\right)$, one gets

$$
\begin{aligned}
\frac{\lambda_{c}}{\alpha_{i}}= & \frac{T_{\perp i}}{T_{\| i}}\left[3+3 \frac{\theta_{\perp}^{3}}{\theta_{\|}^{2}}-\frac{1}{2} \frac{\left(\theta_{\perp}-\theta_{\|}\right)^{2}}{\theta_{\|}^{2}\left(1+\theta_{\|}\right)^{2}}\right. \\
& \left.\times\left(4 \theta_{\perp}+4 \theta_{\|}^{2}+5\left(\theta_{\perp}+1\right) \theta_{\|}\right)\right] \\
& -\frac{3}{2 \theta_{\|}\left(1+\theta_{\|}\right)}\left[\left(\theta_{\perp}+\theta_{\|}\right)^{2}+2 \theta_{\|}\left(1+\theta_{\perp}^{2}\right)\right] .
\end{aligned}
$$

Proceeding as in [13], retaining the contribution of the above quadratic terms to the pressure balance, leads one to supplement a nonlinear contribution to Eq. (11) that becomes

$$
\begin{aligned}
& \frac{\partial u}{\partial t}=\frac{2}{\sqrt{\pi}} \frac{T_{\| i}}{T_{\perp i}} \frac{v_{\| i}}{D} \widehat{\mathcal{K}}_{z}\left\{\Gamma u-\frac{\chi}{\beta_{\perp}}\left(\Delta_{\perp}\right)^{-1} \partial_{z z} u\right. \\
& \left.+\frac{3}{4}\left(\frac{T_{\perp i}}{T_{\| i}}-1\right) \frac{1+F}{1+\theta_{\perp}} r_{L}^{2} \Delta_{\perp} u-\frac{\lambda_{c}}{2\left(1+\theta_{\perp}\right)} u^{2}\right\}
\end{aligned}
$$

Here the integral operator $\widehat{\mathcal{K}}_{z}$ reduces in Fourier representation to $\left|k_{z}\right|$ and $\chi=$ $1+\frac{\beta_{\perp}-\beta_{\|}}{2}$. Furthermore, within the present approximation, $u$ coincides with $\widetilde{B}_{z} / B_{0}$.

Equation (29) extends the result of [13, 21] valid for cold electrons. As in the latter case, this equation is a gradient type equation,

$$
\frac{\partial u}{\partial t}=-\widehat{\mathcal{K}}_{z} \frac{\delta F}{\delta u}
$$

for which the free energy (written in dimensionless variables)

$$
F=\int\left\{\frac{1}{2}\left[-\Gamma u^{2}+\left(\partial_{z} u\right)^{2}+u \Delta_{\perp}^{-1} \partial_{z z} u\right]+\frac{1}{3} \lambda_{c} u^{3}\right\} d \mathbf{r}
$$


is unbounded from below due to the integral $\int \lambda_{c} u^{3} d \mathbf{r}$. This leads to a blow-up behavior, associated with a subcritical bifurcation [13], [14]. Saturation at large values of the amplitude and formation of stationary structures requires additional nonlinear effects such as the influence of resonant particles on the nonlinear coupling [22]. Equation (29) that does not include saturation processes is not suitable to address the question of the reduction of the temperature anisotropy by the development of the mirror instability mentioned in [3]. This effect is reproduced by the quasi-linear theory [19], and was also studied in the context of the so-called FLRLandau fluid model that, like the present asymptotics, retains a linear description of the Landau resonance and of FLR effects, but includes all the hydrodynamic nonlinearities and does not a priori prescribe a pressure balance condition. It was observed in this case that during the saturation phase, the mean temperatures rapidly evolve in a way as to reduce the distance to threshold [20].

As demonstrated in [13, 14], the sign of the nonlinear coupling $\lambda_{c}$ defines the type of the mirror structures, namely holes $\left(\lambda_{c}>0\right)$ or humps $\left(\lambda_{c}<0\right)$, near threshold. This sign is strongly dependent on the equilibium distribution function [23]. It is nevertheless of interest to consider the case where both ions and electrons have a bi-Maxwellian distribution function. It turns out that the sign of $\lambda_{c}$ can then be determined analytically in a few special cases.

(i) Limit $\theta_{\|} \ll \theta_{\perp}$ :

$$
\frac{\Lambda_{c}}{n_{0} T_{\perp i} a_{i}}=\frac{\theta_{\perp}^{2}}{\theta_{\|}}\left(\frac{T_{\perp e}}{T_{\| e}}-\frac{3}{2}\right)>0 .
$$

(ii) Equal anisotropies $\left(\theta_{\perp}=\theta_{\|}\right)$

$$
\begin{aligned}
\Lambda_{c}= & n_{0}\left(T_{\perp i}+T_{\perp e}\right)\left(3 a^{2}-4 a+1\right) \\
& -n_{0}\left(T_{\perp i}+T_{\perp e}\right)(1-a)=3 a \frac{B_{0}^{2}}{8 \pi}>0 .
\end{aligned}
$$

(iii) Isotropic electron temperature: The coefficient $\Lambda_{c}$ can be rewritten in the form

$$
\Lambda_{c}=n_{0}\left(a_{i}-1\right)\left\{T _ { \perp i } \left(\left(3 a_{i}-1\right)\right.\right.
$$




$$
\begin{aligned}
& \left.+c_{i}\left[\frac{1}{2}\left(1+c_{i}\right)\left(\alpha_{i}-1\right)+2-3 a_{i}\right]\right) \\
& \left.+T_{e} c_{e}\left[\frac{1}{2}\left(1+c_{e}\right)\left(a_{i}-1\right)+1\right]\right\}+\frac{B_{0}^{2}}{8 \pi} .
\end{aligned}
$$

Furthermore, at threshold,

$$
\frac{1}{2} n_{0}\left(a_{i}-1\right)\left[T_{\perp i}\left(2-c_{i}\right)+T_{\perp e} c_{e}\right]=\frac{B_{0}^{2}}{8 \pi}>0 .
$$

Hence, we simultaneously have two inequalities $a_{i}>1$ and $T_{\perp e} c_{e}>T_{\perp i}\left(c_{i}-2\right)$. Therefore,

$$
\begin{aligned}
\Lambda_{c}= & n_{0}\left(a_{i}-1\right)\left\{T _ { \perp i } \left(\left(3 a_{i}-1\right)\right.\right. \\
& \left.+c_{i}\left[\frac{1}{2}\left(1+c_{i}\right)\left(a_{i}-1\right)+2-3 a_{i}\right]\right) \\
& \left.+T_{e} c_{e}\left[\frac{1}{2}\left(1+c_{e}\right)\left(a_{i}-1\right)+1\right]\right\} \\
& +\frac{1}{2} n_{0}\left(a_{i}-1\right)\left[T_{\perp i}\left(2-c_{i}\right)+T_{\perp e} c_{e}\right] \\
= & n_{0}\left(a_{i}-1\right)\left\{T _ { \perp i } \left(3 a_{i}\left(1-c_{i}\right)\right.\right. \\
& \left.+c_{i}\left[\frac{1}{2}\left(1+c_{i}\right)\left(a_{i}-1\right)+\frac{3}{2}\right]\right) \\
& \left.+T_{e} c_{e}\left[2+\frac{1}{2}\left(1+c_{e}\right)\left(a_{i}-1\right)\right]\right\},
\end{aligned}
$$

which is positive, because $1-c_{i}=c_{e}=\left(1+\theta_{\|}\right)^{-1}>0$ and $a_{i}>1$.

(iv) More general conditions: A numerical approach was used in this case. Figure 1 displays, for typical values of the parameters (taken here as $\theta_{\perp}=1, a_{i}=1.1$ and $\beta_{\perp i}=10$ ), the distance to threshold $\Gamma$ (dashed line) given by Eq. (21) and the non-dimensional nonlinear coupling coefficient $\lambda=\Lambda /\left(n_{0} T_{\perp i}\right)$ (solid line), where $\Lambda$ is given by Eq. (27), as a function of $\theta_{\|}$. This graph is typical of the general behavior of these functions and shows that they are both decreasing as $\theta_{\|}$increases, with $\lambda$ possibly reaching negative values, but only below threshold. In order to show that the value $\lambda_{c}$, given by Eq. (28), of $\lambda$ at threshold is positive in a wider range of parameters, we display in Fig. 2 , as a function of $\beta_{\perp i}$ for $\theta_{\perp}=0.2$ (solid line), $\theta_{\perp}=1$ (dotted line) and $\theta_{\perp}=5$ (dashed line), the quantity $\min \left(\lambda_{\mathrm{c}}\right)$ 


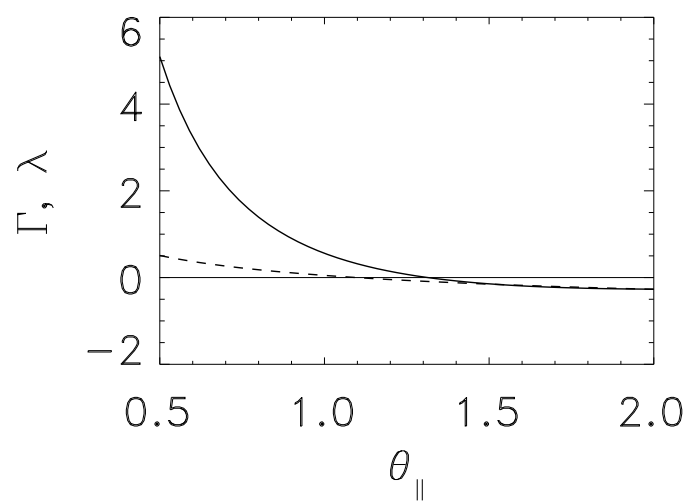

FIG. 1: Fig. 1. Variation with $\theta_{\|}$of the distance to threshold $\Gamma$ given by Eq. (2) (dashed line) and of the normalized nonlinear coupling coefficient $\lambda$ (solid line) evaluated from Eq. (27) for $\theta_{\perp}=1, a_{i}=1.1$ and $\beta_{\perp i}=10$.

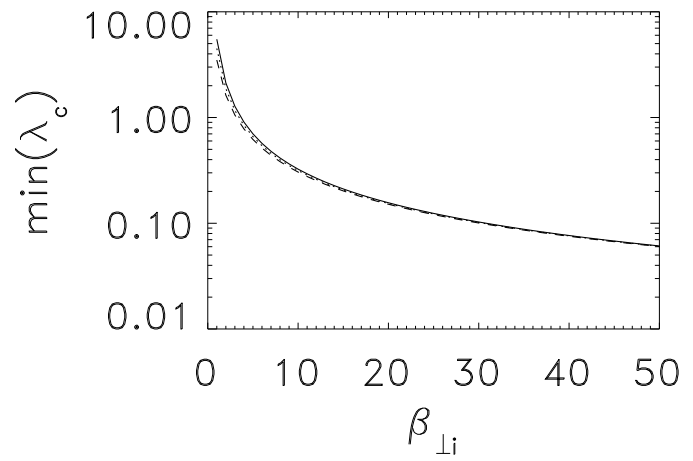

FIG. 2: Fig. 2. Variation with $\beta_{\perp i}$ of the minimum $\min \left(\lambda_{c}\right)$ of the normalized nonlinear coupling coefficient taken in an interval of values of $a_{p}$ between 0 and $a_{p 1}\left(\beta_{\perp i}\right)$, defined such that the threshold is obtained for a value of $\theta_{\|}$equal to 100 , for $\theta_{\perp}=0.2$ (solid line), $\theta_{\perp}=1$ (dotted line) and $\theta_{\perp}=5$ (dashed line).

obtained after minimizing $\lambda_{c}$ in an interval of values of $a_{p}$ between 0 and $a_{p 1}\left(\beta_{\perp i}\right)$. The latter quantity is arbitrarily defined such that the threshold is obtained for a value of $\theta_{\|}$equal to 100 . This graph shows that $\min \left(\lambda_{c}\right)$ varies little with $\theta_{\perp}$ but is very sensitive to $\beta_{\perp i}$. As the latter parameter is increased, $\min \left(\lambda_{c}\right)$ decreases but remains always positive. Although this numerical observation is not a rigorous 
proof, it convincingly shows that $\Lambda>0$ in the parameter range of physical interest.

\section{STATIONARY NONLINEAR STRUCTURES}

Substituting the explicit expressions of the gyrotropic pressures in terms of the magnetic field amplitude given in the Section IV, within the equation for the balance of forces

$$
\begin{aligned}
& -\nabla\left(p_{\perp}+\frac{B^{2}}{8 \pi}\right)+\left[1+\frac{4 \pi}{B^{2}}\left(p_{\perp}-p_{\|}\right)\right] \frac{(\mathbf{B} \cdot \nabla) \mathbf{B}}{4 \pi} \\
& +\mathbf{B}(\mathbf{B} \cdot \nabla)\left(\frac{p_{\perp}-p_{\|}}{B^{2}}\right)=0
\end{aligned}
$$

leads to a closed system that seems overdetermined due to the divergenceless condition $\nabla \cdot \mathbf{B}=0$. In fact, it can be checked, after some algebra using the explicit expressions (20]21) and (24,25), that the projection of Eq. (32) on the magnetic field vanishes identically, thus reducing the system to three equations for three unknowns. These equations can be useful for finding, possibly numerically, stationary profiles of three-dimensional finite-amplitude stationary mirror structures. Note that Eq. (32) differs from the Grad-Shafranov equation [24, 25] in that the parallel and perpendicular pressures are here prescribed functions of the magnetic field amplitude. A main issue concerns the existence of stable subcritical solutions, a question that is beyond the scope of this letter and will be addressed in forthcoming works. Such structures are reported by satellite observations [26, 27] and are also expected from the subcritical character of the mirror instability [14]. Equilibrium solutions were computed in one-space dimension in [17], where they lead to discontinuous profiles. Their regularization would require that FLR corrections be retained. These additional contributions are known from the linear kinetic theory but their extension to the finite-amplitude case remains a challenging problem.

This work was supported by the CNRS PICS programme 6073 and RFBR grant 12-02-91062-CNRS_a. T.P. and P.L.S. benefited from support from INSU-CNRS Programme National PNST. The work of E.K. was also supported by the RAS 
Presidium Program "Fundamental problems of nonlinear dynamics in mathematical and physical sciences", Grant NSh 7550.2006.2 and by the French Ministère de l'Enseignement Supérieur et de la Recherche.

[1] P.L. Sulem, AIP Conf. Proc., 1356, 159, (2011).

[2] V. Génot, E. Budnik, C., Jacquey, I. Dandouras, I., and E. Lucek, Adv. Geosci., vol. 14: Solar Terrestrial (ST), edited by M.Duldig, p. 263, (World Scientific, 2009)

[3] A.A. Vedenov and R.Z. Sagdeev, Plas. Phys. \& Problem of Cont. Therm. React., Vol. III, ed. M.A. Leontovich, 332 (Pergamon Press, NY, 1958).

[4] Š. Štverák, P. Trávníček, M. Maksimovic, E. Marsch, A. N. Fazekerley and E. E. Scime", J. Geophys. Res. 113, A03103 (2008).

[5] T.H. Stix, The Theory of Plasma Waves, McGraw-Hill, 1962.

[6] F.G. E. Pantellini and S. J. Schwartz, J. Geophys. Res. 100, 3539-3549 (1995).

[7] V. Génot, S. J. Schwartz, C. Mazelle, M. Balikhin, M. Dunlop and T. M. Bauer, J. Geophys. Res. 106, 21611-21622 (2001).

[8] O.A. Pokhotelov and M. A. Balikhin and H. St-C. K. Alleyne and O. G. Onishchenko, J. Geophys. Res. 105, 2393-2401 (2000).

[9] P. Hellinger, Phys. Plasmas 14, 082105 (2007).

[10] E.A. Kuznetsov, T. Passot and P. L. Sulem, Phys. Plasmas, 19, 090701 (2012) .

[11] A.N. Hall, J. Plasma Physics 21, 431, (1979).

[12] O.A. Phokhotelov, M.A. Balikhin, R.Z. Sagdeev, and R.A. Treumann, J. Geophys. Res. 110, A10206 (2005).

[13] E.A. Kuznetsov, T. Passot, and P.L. Sulem, Phys. Rev. Lett. 98, 235003 (2007).

[14] E.A. Kuznetsov, T. Passot, and P.L. Sulem, JETP Letters 86, 637-642 (2007).

[15] V.E. Zakharov and E.A. Kuznetsov, Physics - Uspekhi 55, 535 (2012).

[16] O.D. Constantinescu, J. Atm. Solar-Terrestrial Phys. 64, 645-649 (2002).

[17] T. Passot, V. Ruban and P. L. Sulem, Phys. Plasmas 13, 10231 (2005). 
[18] A. Hasegawa, Phys. Fluids 12, 2642, ,(1969).

[19] V.D. Shapiro and V.I. Shevchenko, Zh. Eksp. Teor. Fiz. 45, 1612 (1963) [J. Sov. Phys. JETP 18, 1109 (1964)].

[20] D. Borgogno, T. Passot, and P.L. Sulem, Nonlin. Process. Geophys., 14, 373-383 (2007).

[21] F. Califano, P. Hellinger, E. Kuznetsov, T. Passot, P.L. Sulem, and P. Travnicek, J. Geophys. Res. 113, A08219, (2008).

[22] T. Passot, P.L. Sulem, E. Kuznetsov, and P. Hellinger, AIP Conf. Proc., 1188, 205 (2009).

[23] P. Hellinger, E. Kuznetsov, T. Passot, P.L. Sulem, and P. Trávníček, Geophys. Res. Lett. 36, L06103 (2009).

[24] H. Grad and H. Rubin, Proc. 2nd Conf. on the Peaceful Use of Atomic Energy, 31, 190 (IAEA, Geneva, 1958).

[25] V.D. Shafranov, Zh. Eksp. Teor. Fiz. 33, 710 (1957) [J. Sov. Phys. JETP 8, 545 (1958)]; Rev. Plasma Phys. 2, 103 (Consultant Bureau, New York, 1966).

[26] J. Soucek, E. Lucek and I. Dandouras, J. Geophys. Res. 113, A04203 (2007).

[27] V. Génot, E. Budnik, P. Hellinger, T. Passot, G. Belmont, P.M. Trévníček, P.L. Sulem, E. Lucek and I. Dandouras, Ann. Geophys. 27, 601-615 (2009). 